Écrire

l'histoire

\section{Écrire l'histoire}

Histoire, Littérature, Esthétique

15 | 2015

La fin de l'histoire

\title{
La Fin du monde. Essai sur les apocalypses culturelles (extraits)
}

\section{Ernesto De Martino}

Traducteur : Giordana Charuty

\section{OpenEdition \\ Journals}

Édition électronique

URL : http://journals.openedition.org/elh/605

DOI : $10.4000 /$ elh.605

ISSN : 2492-7457

Éditeur

CNRS Éditions

Édition imprimée

Date de publication : 8 octobre 2015

Pagination : 101-108

ISBN : 978-2-271-08822-2

ISSN : $1967-7499$

\section{Référence électronique}

Ernesto De Martino, "La Fin du monde. Essai sur les apocalypses culturelles (extraits) », Écrire l'histoire [En ligne], 15 | 2015, mis en ligne le 08 octobre 2018, consulté le 23 septembre 2020. URL : http:// journals.openedition.org/elh/605; DOI : https://doi.org/10.4000/elh.605 


\section{La Fin du monde. Essai sur les apocalypses culturelles (extraits)}

\section{Extraits présentés et traduits de l'italien par Giordana Charuty}

Lorsqu'il disparaît brutalement en mai 1965, l'anthropologue italien Ernesto De Martino laisse le manuscrit inachevé d'une vaste enquête qui sera publiée douze ans plus tard par l'éditeur Einaudi: La fine del mondo. Contributo all'analisi delle apocalissi culturali [La Fin du monde. Essai sur les apocalypses culturelles] (1977). Une version réduite, et recomposée à partir des dossiers d'archives, de cette recherche méconnue dans le monde intellectuel français paraîtra dans la collection "Translations" des éditions de l'EHESS'. Nous en présentons ici quelques extraits.

De Martino a inscrit son enquête dans la conjecture intellectuelle qui a vu l'intérêt de nombreux ethnologues et historiens se fixer, au milieu des années cinquante, sur les messianismes révolutionnaires du tiers monde. Ainsi, à la suite des travaux de l'africaniste Vittorio Lanternari, De Martino prévoyait de consacrer un chapitre aux rapports entre mouvements apocalyptiques et décolonisation ${ }^{2}$. Mais l'ambition de son projet va bien au-delà. Il s'agit de comparer les ressources culturelles offertes par des formations sociales aussi diverses que le prophétisme apocalyptique du christianisme primitif, les mobilisations millénaristes du tiers monde ou l'émergence du mouvement communiste international, à travers leur manière de penser l'historicité, pour répondre à ce que l'auteur identifie comme un risque anthropologique permanent: la perte de la présence au monde. Ce risque est mis à nu dans les récits cliniques d' "expérience vécue de fin du monde » documentés, entre les années 1920 et les années 1960, par la psychiatrie d'inspiration phénoménologique. Réélaborés en matière ethnographique, ils permettent de reconnaître une apocalypse culturelle "sans eschaton» dans les formes littéraires et plastiques de la modernité esthétique. Une redéfinition de la culture comme ce qui 
préserve de la folie est, ainsi, au cœur des propositions pour une ethnologie réformée qui fixe, du même pas, les exigences analytiques qui devraient s'imposer à tout historien des formations religieuses.

\section{Notes}

1 Sous la direction de Giordana Charuty, Daniel Fabre, Marcello Massenzio.

2 Le numéro 161 d'Archives de sciences sociales des religions (2013) présente ce contexte dans le dossier «Messianismes et anthropologie entre France et Italie», coordonné par Daniel Fabre et Marcello Massenzio.

Le texte publié se présente sous forme de fragments. Nous reproduisons ici la numérotation de la première édition italienne (1977). L'édition française est composée d'une sélection de ces fragments, recomposés à partir des dossiers présents dans les archives démartiniennes. Une équipe de traducteurs en a établi une première version, révisée par Giordana Charuty, Daniel Fabre et Marcello Massenzio. Autorisation de traduction et de publication: éditions Einaudi.

Les notes et les intertitres de ce montage sont de Giordana Charuty.

$<30>$ La fin du monde relève de la physiologie d'une culture ou de la psychopathologie d'une biographie individuelle selon le sens du dynamisme dans lequel ce motif s'insère. Nous sommes dans la physiologie d'une culture quand «la fin du monde» dénote un horizon mythico-rituel au sein duquel le risque d'un monde qui finit, vécu sur le mode privé et incommunicable, est repris et réintégré selon des valeurs intersubjectives et communicables, moyennant une marge de manœuvre pour être au monde ; en revanche, quand le dynamisme est de sens opposé et que les horizons culturels se désarticulent et s'effondrent en se repliant vers le vécu privé et incommunicable d'une «fin» sans reprise efficace, nous sommes dans la sphère psychopathologique individuelle. Dans le premier cas, le jugement revient surtout à l'historien de la culture, dans le second cas essentiellement au psychiatre; mais en raison du lien organique entre le risque individuel et sa reprise culturelle, l'historien ne peut s'abstenir des indications du psychiatre, et le psychiatre ne peut se soustraire - en tant qu'expert du diagnostic des maladies psychiques - à l'exigence d'un jugement historique qui réintègre l'épisode morbide dans la biographie du malade et celle-ci dans un contexte historico-culturel concret.

$<\mathbf{1 0 8}>$ La fin d'un monde donné peut être traitée sous deux angles différents: comme représentation culturelle historiquement déterminée et comme risque anthropologique permanent. Dans le premier cas, le motif apparaît au sein de configurations mythiques qui $y$ font explicitement référence: par exemple, les destructions et régénérations périodiques du monde associées au mythe de l'éternel retour ou bien l'ultime catastrophe de l'Histoire, dans le cadre d'une représentation unilinéaire et irréversible du temps. Comme risque anthropologique permanent, le finir est simplement le risque de ne pouvoir 
être-là en aucun monde culturel possible, la perte de toute présence active au monde, le rétrécissement - jusqu'à l'anéantissement - de tout horizon de disponibilité, l'effondrement de toute projectualité ${ }^{1}$ communautaire guidée par des valeurs. La culture humaine, au sens général, est l'exorcisme solennel de ce risque radical, quelle que soit, peut-on dire, la technique d'exorcisme adoptée ; et si le motif culturel de la fin d'un ordre mondain constitue l'une des modalités historiques de reprise et de délivrance de ce risque, y compris là où cette représentation est absente ou insignifiante, le risque corrélatif est, lui, toujours présent, et la culture se construit justement dans l'effort pour le contrôler, quelle que soit la manière dont le drame est réfléchi dans une élaboration culturelle historiquement déterminée.

\section{Formules cycliques du temps}

$<110>$ La conscience cyclique du temps, qui s'exprime sur le plan religieux par la répétition rituelle d'un mythe des origines et d'un récit de fondation, et sur le plan cosmologique par la théorie de l'éternel retour, constitue, au temps de sa plus grande fécondité, un régime de protection pour médiatiser l'historicité du devenir humain: un régime qui le défend de la tentation toujours présente d'anéantir le devenir dans la paresseuse répétition à l'identique et la pure cyclicité $d u$ retour-à. La répétition rituelle d'un mythe des origines symbolise une sorte d'imitatio naturae: on reprend le revenir-à et on le modèle culturellement pour en faire l'horizon d'ouverture de l'être-au-monde. Le devenir de l'homme qui s'élève contre cette paresse de l'infrahumain continue à être travaillé par elle: l'irréversibilité du temps humain risque de devenir réversible. Reprendre la réversibilité pour la changer de signe: ainsi procède le symbolisme de la répétition rituelle du mythe des origines.
[...] La tradition judéo-chrétienne laisse pénétrer au sein même de la conscience mythico-rituelle la conscience historique du devenir irréversible: l'accent se déplace de l'exemplarité des origines à l'exemplarité du moment central du devenir, du divin au divin incarné (le Christ), de la répétition des origines à la répétition du moment central (la mort et la résurrection du Christ), de la cyclicité des catastrophes à l'attente d'une fin orientée vers une unique direction (le Royaume). À la répétition des origines divines de l'Histoire succède, désormais, la répétition du centre divin-humain de l'Histoire qui renvoie le problème du salut individuel à la fin unique annoncée. Le grand problème de notre époque est celui d'un salut de l'individu dans la société des hommes, à travers une socialisation qui ne soit pas une massification, une bureaucratisation, une automatisation, un technicisme, une idolâtrie de l'État, une divinisation du chef, etc. 


\section{L'apocalypse culturelle chrétienne}

$<272>$ [...] L'apocalypse chrétienne est un mouvement historique qui s'élève au-dessus du monde vécu comme déjà fini, ou de l'attente d'une fin déterminée, ou du nihilisme d'une fin indéterminée toujours imminente, pour déployer en œuvrant dans le monde un témoignage de la fin du monde, pour préparer le jour du Seigneur et instaurer progressivement le monde chrétien. L'apocalypse chrétienne est un mouvement qui fonde un monde, qui crée une civilisation, replace la fin comme crise et la terreur de chaque commencement mondain dans l'horizon protecteur qu'instaure le déploiement des vertus chrétiennes: à travers ce remodelage culturel, la fin comme crise est reprise, surmontée dans la fin comme eschaton en libérant, entre l'une et l'autre, l'espace transitoire d'un monde chrétien en expansion.

[...] Les apparitions de Jésus, depuis celle aux femmes à proximité du sépulcre jusqu'à la dernière à table, puis à l'Ascension, qui clôt le cycle protochrétien, scandent dans l'Évangile les moments d'une grande liturgie funéraire qui offre son modèle pour dépasser la mort et la crise du deuil qui l'accompagne: ce modèle narratif s'achève, après la séparation de l'Ascension, avec la descente de l'Esprit saint qui inaugure l'époque où le mort-ressuscité sera présent chaque jour aux côtés des croyants, jusqu'à la fin du monde, pour leur donner la dynamis missionnaire qui caractérise ce temps.
On peut donc interpréter la genèse $d u$ protochristianisme comme exemplification de la résolution historique d'une crise de deuil: résolution qui transforme Jésus mort dans le Christ ressuscité, le mort-qui-revient de la crise dans le mort-ressuscité présent dans l'Église et, par excellence, dans le banquet eucharistique, jusqu'à ce que le déjà advenu de la promesse soit accompli à travers l'élan missionnaire. Cela nous invite à approfondir la thèse de Mort et lamentations rituelles dans le monde antique et autorise à interpréter le christianisme comme un grand rituel funéraire où le traitement exemplaire d'une mort permet de traiter les différents mourir historiques, à travers une pédagogie du détachement et du dépassement de ce qui meurt ${ }^{2}$. (Ce qui ne pouvait se faire que pour autant que le mort était l'«oint», c'est-à-dire l'homme-Dieu.) Cette résolution était aussi présente dans les rituels funéraires du monde antique, mais selon un dispositif différent puisque leur manquait, justement, ce qui est au centre du christianisme: l'exemplarité historique d'un passage de la crise d'un mort-qui-revient à la foi en un mort-ressuscité ; de la foi en un mort-ressuscité à la présence de celui-ci dans la communauté des fidèles ; et de sa présence dans la communauté des fidèles au devoir de témoigner ardemment pour lui dans une vie chrétienne contenue dans l'horizon de sa promesse. 


\section{L'héritage mythique du marxisme}

$<248>$ La religion comme reconnaissance de l'homme à travers un chemin indirect et long est une formulation qui a donné lieu à des équivoques. Dans une logique de combat pour la transformation de la réalité, les marxistes ont vu là une invitation faite aux hommes à se reconnaître eux-mêmes à travers le chemin direct et bref d'une réappropriation de leur propre faire historique, ce qui pourra advenir dans un autre régime d'existence, lorsque ce faire leur sera pleinement restitué dans une société sans classe, où la raison contrôle la nature au même titre que la vie en société. Or, il faut noter:

$1^{\circ}$ Que la réappropriation complète du faire humain est un produit de la pensée mythique et qu'elle équivaut pratiquement au retour à Dieu et à la fin de l'histoire. L'aliénation est un risque inhérent à la condition humaine, seul est possible le progrès dans la réappropriation de l'homme (c'est le problème actuel du passage de la société capitaliste à la société socialiste) ; dans les sociétés socialistes ou communistes comme dans toute autre société historique, les risques d'aliénation ne sont jamais éliminés, les contradictions et les conflits revêtent une nouvelle forme. L'idée d'une histoire jusque-là inhumaine qui, grâce au socialisme, deviendra intégralement humaine appartient évidemment à l'héritage mythique du marxisme, comme cadre d'action destiné à mobiliser les masses.

$2^{\circ}$ Que si la réappropriation complète de l'homme est un héritage mythique, l'idée selon laquelle les civilisations religieuses vivraient en pleine aliénation l'est tout autant. Toutes les civilisations se réapproprient, en quelque sorte, le faire humain, puisque l'expérience que "ce que je fais c'est moi qui le fais», que «cet agir m'appartient», détermine la civilisation elle-même. La religion est donc, elle aussi, un moyen de combattre l'aliénation, le seul possible dans certaines conditions de développement socio-économique. L'analyse historique des symbolismes mythico-rituels doit par conséquent servir à expliquer comment, dans certaines conditions, s'affirme un secteur, même très étroit, de l'agir humain reconnu comme tel, le «je suis en train de faire ce que je fais » et le «ce que je suis en train de faire est mon ouvrage».

$<260.3>$ Dans la vie religieuse de l'humanité, le motif de la fin du monde est présent en différents contextes eschatologiques, comme régénération périodique du monde ou comme libération définitive des maux inhérents à l'existence mondaine: pensons, par exemple, au nouvel an des civilisations agricoles ${ }^{3}$, aux mouvements apocalyptiques des peuples coloniaux aux XIX ${ }^{\mathrm{e}}$ et $\mathrm{XX}^{\mathrm{e}}$ siècles ${ }^{4}$, à l'histoire du salut dans la tradition judéo-chrétienne, aux multiples millénarismes dont est constellée l'histoire religieuse occidentale ${ }^{5}$. À l'opposé de cette perspective eschatologique, le motif de la fin est aujourd'hui présent dans la culture occidentale, en dehors de tout horizon religieux de salut, comme catastrophe désespérée du mondain, du domestique et du familier, du sensé et de l'utile: une catastrophe qui relate avec un soin méticuleux, voire obses- 
sionnel, la désagrégation du monde ordonné, l'altération du domestique, la perte de la familiarité et du sens, l'indisponibilité pratique du monde. Certes, la conjoncture culturelle de l'Occident ne se réduit pas à ce thème désespérant et désespéré, elle oppose une diversité de réponses à cet appel mortel qui peut simplement l'effleurer, la toucher ou l'as- saillir comme un vent de tempête ; toutefois, l'abandon sans retour à l'expérience vécue de la fin constitue indéniablement une disposition propre à notre époque, ce dont peuvent témoigner nombre de documents tirés des mœurs, de la littérature, des arts figuratifs, de la musique et enfin de la philosophie elle-même.

\section{Les réveils de Proust}

$<305.5>$ La recherche proustienne du temps perdu n'exprime pas tant le libre déploiement de l'énergie recréatrice et transfiguratrice de la poésie (même si elle scintille parfois dans le courant de remémoration), mais plutôt la problématisation d'une puissance primordiale, qui est aux racines de toute vie culturelle et de tout être-au-monde selon des valeurs intersubjectives, c'est-à-dire le pouvoir de rappeler sans cesse le passé à divers niveaux d'engagement et de conscience, le pouvoir de ressaisir sans cesse ce passé dans un horizon d'action créatrice de valeur. Proust est l'expert littéraire, parfois le poète, de la crise du rapport moimonde: il redescend au niveau où les choses, les personnes, son propre corps, se font tombes anonymes, voire fosses communes du passé, mais il y redescend pour surprendre l'émergence de cette remémoration primordiale qui donne sens au monde et qui assure la liberté et la disponibilité d'un arrière-plan domestique communautaire. La fameuse résurrection de Combray à partir de la tasse de thé signifie que, par une morne journée $d$ 'hiver et avec la perspective d'un triste lendemain, lorsque ce lien mémoriel semble se briser, que le monde se déco- lore et que le temps se rétracte, même une tasse de thé a le pouvoir d'inverser les signes, de susciter la chaude vague affective qui renferme les jours cosmogoniques de Combray, dont le parfum retisse, en se diffusant, la trame d'un monde disponible. Dans les «réveils» sur lesquels s'ouvre la Recherche, Proust saisit avec une grande finesse d'analyse les chemins parcourus vers l'être-là à partir d'un risque radical de chute dans le néant. Tenir ainsi en cercle autour de soi, dans son sommeil, «le fil des heures, l'ordre des années et des mondes», risquer de rompre ce fil dans le réveil et de se sentir plongé dans une désorientation totale, se trouver "plus dénué qu'un homme des cavernes» et traverser en une seconde «des siècles de civilisation" en retrouvant dans l'image confusément entrevue de la lampe à pétrole ou de la chemise à col rabattu un premier ancrage de mémoires culturelles porteuses d'orientation qui vaut comme billet d'entrée dans la familiarité historique du monde, recomposer ainsi l'historicité du moment en remontant le cours «des choses, des pays, des années», et revivre - par l'évocation de mémoires abritées dans son propre corps - les 
autres chambres dans lesquelles on a dormi - les chambres de Combray, de Tansonville, de Balbec -, tout cela éclaire les replis secrets de l'entière cosmogonie qui, à chaque instant, s'accomplit en nous, bien que ce mouvement soit masqué par l'évidence et la familiarité de l'arrière-plan qui permet à notre liberté de s'affirmer. Ajoutons qu'évidence et familiarité dépendent, à leur tour, de l'existence de telles mémoires latentes, qui nous relient tacitement au fil des heures, à la succession ordonnée des années et des mondes jusqu'à l'homme des cavernes, des mémoires qui abritent non seulement notre histoire familiale mais aussi, au long des générations et des âges, toutes les œuvres issues de projets communautaires qui ont fait du monde la patrie culturelle de l'homme. Ce n'est que dans la déstructuration de ce cours, dans le risque de l'inversion de sens, que commence à s'effriter le roc de la familiarité.

\section{Être présent au monde}

<355> Le «monde» est toujours donné comme totalité communautaire à reprendre dans la spécificité d'une forme singulière de valorisation, il est toujours mis à distance comme monde de la quotidienneté pour pouvoir tracer dans ce vécu plus ou moins anonyme et socialisé l'empreinte intime et personnelle d'une initiative individuelle, créatrice de valeurs. Mais la datitée ${ }^{6}$ la quotidienneté, l'évidence du monde, ne sont possibles que parce qu'elles représentent la fidélité immédiate à des initiatives fruit de générations passées, ou de notre propre passé et de tout ce qui $s^{\prime} y$ rattache dans notre biographie culturelle: et si elles constituent ce sol et cette patrie sur lesquels peut s'édifier la tâche du moment, c'est parce que seul ce passage par la domestication anonyme du monde nous rend disponibles pour la poursuivre à travers l'incessante reprise de «nos» choix propres, dans leur singularité. Un individu ne peut «recommencer» certains aspects du monde - et il les recommence toujours comme s'il était le premier homme à devenir homme pour la première fois - que si l'ensemble des autres aspects forment momentanément un arrière-plan, et si cet arrière-plan est implicitement porteur d'un sens humain, d'un travail d'humanisation inscrit dans l'évidente familiarité du monde ambiant, d'un témoignage essentiel d'absence de solitude, d'humble mise en œuvre socialisée dans l'espace et le temps. Datité et reprise sont unies par un lien dialectique dont le relâchement annonce l'effondrement du monde et de la présence qui s'y rapporte: lorsque l'humble mise en œuvre socialisée perd ce caractère $\mathrm{d}^{\prime}$ «arrière-plan» d'où émerge mon initiative, lorsque cet arrière-plan devient un problème et que tout est remis en cause sans laisser de marge au dépassement ni de «point d'appui» pour «le saut originaire», alors le monde perd son aspect de "familiarité», de "patrie de l'agir», de «sol» stable pour œuvrer de manière efficace, et il devient ce qui dépossède et envahit le domaine privé. 


\section{Notes}

1 La projectualité désigne le moment d'élaboration d'une valorisation du monde comme processus communautaire.

2 Dans Morte e pianto rituale nel mondo antico: dal lamento pagano al pianto di Maria (Turin, Einaudi, 1958), De Martino analysait exclusivement la christianisation des lamentations funèbres à travers le thème culturel de la Mater dolorosa et des lamentations de la Vierge. Le thème du «retour du mort» est documenté par l'ethnographie dans toute l'Europe chrétienne. Son analyse a été renouvelée par l'anthropologie de la mort qui s'est développée dans les années 1980, en reprenant le questionnement démartinien sur les composantes de la personne.

3 Allusion à l'étude de Vittorio LANTERnARI, $L a$ grande festa. Storia del Capodanno nelle civiltà pri- mitive [La grande fête. Histoire du nouvel an dans les civilisations primitives], Milan, Il Saggiatore, 1959.

4 Id., Les Mouvements religieux de liberté et de salut des peuples opprimés [ $1^{\text {re }}$ éd. en italien 1960], trad. par Robert Paris, F. Maspero, 1962.

5 Norman CoHn, Les Fanatiques de l'Apocalypse. Millénaristes révolutionnaires et anarchistes mystiques au Moyen Âge [1 ${ }^{\text {re }}$ éd. en anglais 1957], éd. revue et augmentée, Payot (Bibliothèque historique), 1983.

6 La datité est, ici, empruntée au vocabulaire technique de l'ontologie heideggérienne pour désigner un mode d'être. 